\title{
Modeling and performance analysis of IP access interface in optical transmission networks with packet switching
}

\author{
M. Lackovic · C. Bungarzeanu
}

C Springer Science + Business Media, LLC 2006

\begin{abstract}
The article analyzes the influence of the Internet Protocol (IP) access interface on the packet loss probability and delay times in the optical packet switched network. The network and node model have been proposed, and the structure of the IP access interface, including assembler and holder, have been included in the analysis. It has been shown that the increase of the maximum optical packet sizes, as well as use of the holding feature as contention resolution mechanism, decrease the packet loss probability, but introduce delays at the optical network access points. Modeling and analysis were based on the discrete event simulation assuming self-similar traffic sources. IP packet lengths were modeled using empirical data.
\end{abstract}

Keywords Optical network $\cdot$ Optical packet switching $\cdot$ Access interface $\cdot$ Network modeling · Node modeling $\cdot$ Simulation

\section{Introduction}

The Wavelength Division Multiplexing (WDM) technology boosted optical fiber transmission by accommodating the ever increasing capacity demands. However, optical transmission networks made a small step from the first point-to-point systems in terms of reconfigurability and manageability. Photonic layer still serves solely as a transmission medium, which is on the bottom of the inefficient and unscalable protocol stack. Routing, protection and restoration, and traffic/quality of service (QoS) management mechanisms are spread across several stack layers,

M. Lackovic $(\triangle)$

Ericsson Nikola Tesla, Krapinska 45, p.p. 93, HR-10000 Zagreb, Croatia

e-mail: marko.lackovic@ericsson.com

C. Bungarzeanu

Telecommunication Laboratory, EPFL, CH-1015 Lausanne, Switzerland

e-mail: cristian.bungarzeanu@epfl.ch

${ }^{1}$ This work has been conducted while the author was affiliated with the Telecommunication Laboratory, Swiss Federal Institute of Technology (EPFL-STI-ITOP-TCOM), Lausanne, Switzerland 
Fig. 1 Optical transmission network structure

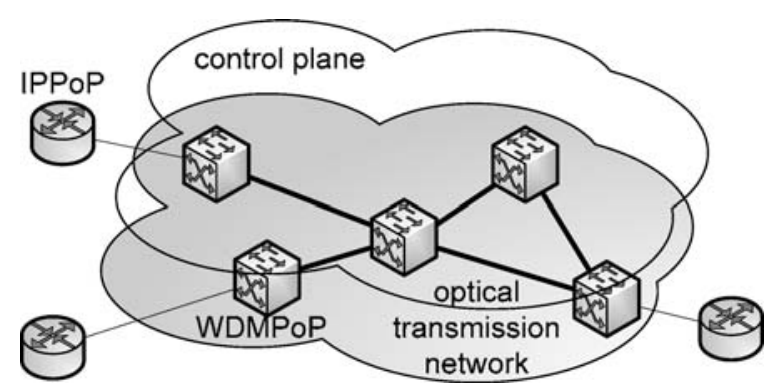

resulting in overlapping protocol functionalities and waste of resources due to large communication overheads.

The idea of "intelligent" optical layer started to emerge in the context of decreasing operators' revenues due to low price of best-effort communication, and increasing user demands for capacity accompanied by the quality of service guarantees. The user stack should be consolidated to provide a simple and robust communication between the photonic layer and the IP as its primary client. The intermediate protocol functionalities can migrate either to the upper, IP layer, or to the lower, photonic layer. The resulting paradigm, where IP entities communicate directly using photonic layer as a dynamic service provider for packet based communication, is often denoted as the optical packet switching (OPS) [1]. The interface between the IP and the photonic layer is a main issue in the future optical network design. This includes transformation of ingress IP packets in optical packets, as well as contention resolution schemes in the IP/photonic interfaces.

The paper consists of six chapters, staring with the general introduction to optical packet switching. Used node and network architectures are explained in the second chapter. Two node types are introduced and their functionality is decomposed into submodules and explained in details. Optical packet structure is described in the third chapter, while the aggregation of the IP datagrams into optical packets is elaborated in the fourth chapter. The fifth chapter gives an overview of obtained results including performance evaluation of the aggregation and the holding mechanism. The paper is concluded with the sixth chapter.

\section{Node and network architectures}

The general idea of this work is to propose and analyze an optical packet switching model able to support the network planning procedure and the network performance analysis, with the focus on the IP access interface. The model should thus address the optical packet switching as well as the functionalities of network clients, primarily IP. Node and network models were developed using the object-oriented paradigm of the Cosmos tool [2].

The network is structured using two node types, namely IP Point of Presence (IPPoP) node and WDM Point of Presence (WDMPoP) node, as shown in Fig. 1. IPPoP and WDMPoP are generic nodes implementing optical network (in WDMPoP node) and IP network (in IPPoP node) functionality.

\section{A. IPPoP}

IPPoP node implements the basic IP layer functionality needed to transmit IP packets, hence enabling the network performance analysis. The basic functionality includes initial definitions of traffic demands between each pair of nodes, which serve as the input data for the dimensioning 


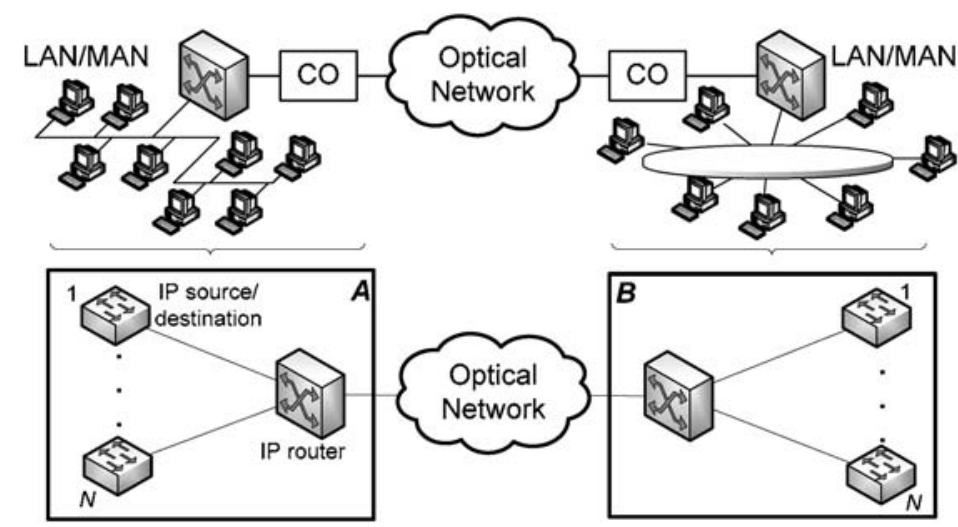

Fig. 2 IPPoP node functionality
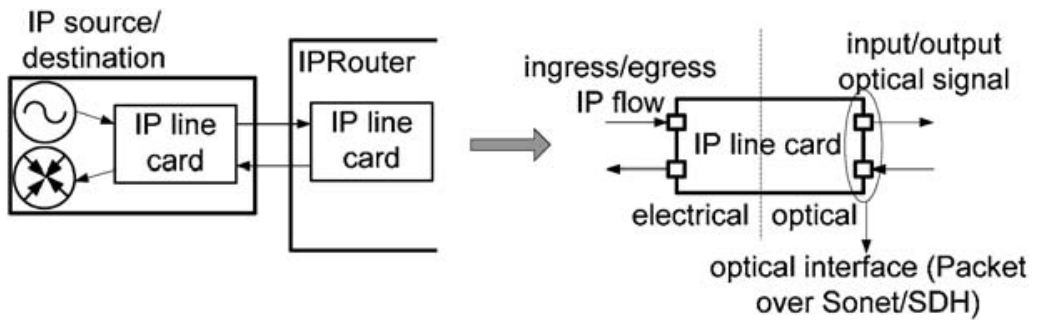

Fig. 3 Line card model

procedure. This data is used for parameterization of the aggregated traffic sources in the simulation phase.

IPPoP nodes are modeled as IP entities, which represent clients of the optical layer, such as traffic generators and IP routers. These clients are characterized in this work by the capacity required from the optical network. An IPPoP node can represent a whole range of possible IP entities from one computer to a whole metropolitan network comprising large number of local area networks and users. Figure 2 clarifies the case where IPPoP node substitutes local area networks/metropolitan area networks (LAN/MAN), e.g. a whole city. The chain of operators (CO) interconnects the clients (IPPoP node) with the optical networks. We will assume in this work that the IPPoP nodes are connected directly to the optical network.

The IPPoP node dimensioning is determined by the traffic demand capacity between the selected node (node $A$ ), and all other IPPoP nodes in the network (node $B$ ).

Figure 2 illustrates the IPPoP node structure. It includes sources and destinations of IP traffic (denoted as IPSD), along with the IP router. Each IPSD module models one communication between the selected node and some other IPPoP node. IPSD modules are connected to the optical network via routers using line cards (IPLC), the structure of which is shown in Fig. 3. The line cards represent an electrical-optical interface converting the electrical signal to the optical form. A router in this model acts as a passive concentration point implementing the interface between IP sources/destinations and WDMPoPs.

Line cards in the IPSDs and routers have Packet over Sonet (PoS) interfaces, which are currently a dominating solution for broadband optical communication. The number of IPSDs is determined by the demand capacity that has to be supported and the PoS interface capacity. The 


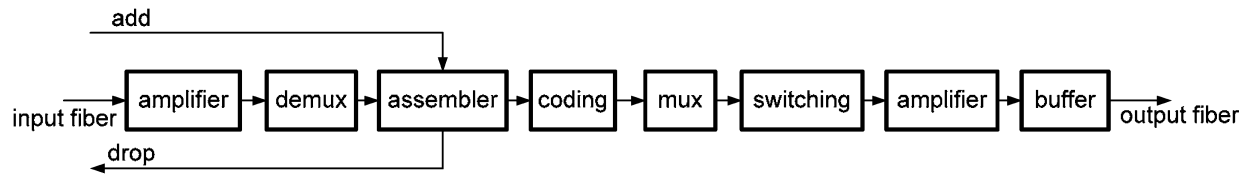

Fig. 4 WDMPoP functional decomposition

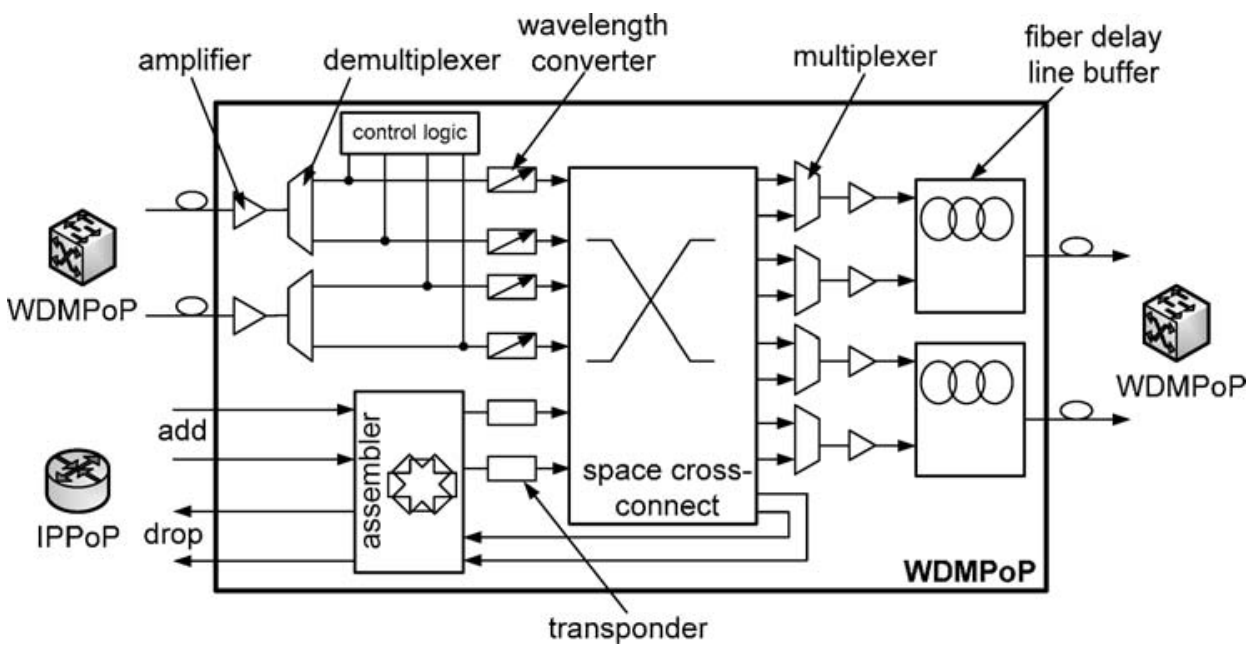

Fig. 5 General WDMPoP node structure

number of PoS line cards in the node $i$ is equal to

$$
N_{P o S}(i)=\sum_{0 \leq i<n, j \neq A} N_{S D}(i, j), N_{S D}(i, j)=\left\lceil\frac{T(i, j)}{C_{P o S}}\right\rceil,
$$

where $T(i, j)$ denotes a capacity of traffic demands between IPPoP nodes $i$ and $j, C_{P o S}$ denotes a PoS interface capacity and $n$ stands for the number of IPPoP nodes. $N_{S D}(i, j)$ is the number of IPSD modules supporting the node $i$ to node $j$ communication. Symmetrical communication capacities between all node pairs are assumed as a consequence of equal transmitter and receiver line card capacities.

\section{B. WDMPoP}

WDMPoP node, the functional decomposition of which is shown in Fig. 4, and the architecture in Fig. 5, primarily performs optical packet switching. In Fig. 4, the term coding stands for the tunable wavelength conversion.

The key component for this work is the assembler, as it implements the interface between the IPPoP and the WDMPoP node. This node architecture can be classified as an output buffered optical packet switching node with space switching and full wavelength conversion. Optical buffers are based on fiber delay lines (FDLs).

The input traffic is the WDM multiplexed signal from other WDMPoP nodes, or a baseband Sonet/SDH framed signal from the IPPoP assigned to the WDMPoP (add flows). Each WDMPoP 
Fig. 6 Control logic structure

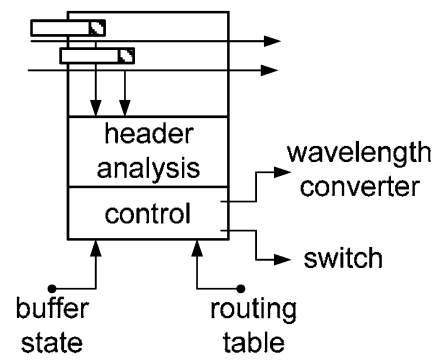

node hence serves as an edge or a core optical packet switch as it has ingress traffic flows from both the core (multiplexed signal) and the access (baseband signal) networks. WDM signals arriving to the inputs are demultiplexed and analyzed on the packet level in the control logic part (Fig. 6).

The header data along with the buffer state and routing table information (Fig. 6) determines the configuration of the coding and switching section. The coding section comprises tunable wavelength converters controlled by the control logic module. The output wavelength depends on the egress packet scheduling. This includes the choice of the output port (link) determined by the routing table and the state of the port or its occupancy. If all the channels (wavelengths) on the selected output port are occupied, the packet has to be rejected if no contention resolution scheme apart from wavelength conversion is used, or buffered/deflected as a part of the contention resolution scheme [3]. The choice of the wavelength and fiber delay line depends on the implemented buffer mechanism and/or QoS support. A through analysis of the buffering schemes is provided in [4], while the possibilities for QoS packet differentiation in the optical domain are analyzed in [5].

\section{IPPoP - WDMPoP interface}

A baseband optical fiber, with Sonet/SDH framing, serves as a connection between IPPoP and WDMPoP nodes. This enables the same type of interface for the communication between IP sources and destinations (e.g. LAN egress switch) and routers, as well as between routers and WDMPoPs.

The PoS interfaced cards for the IPPoP to WDMPoP communication are denoted as PoSW. The number of PoSW line cards depends on the total egress traffic from one IPPoP node as it is assumed that each WDMPoP has one assigned IPPoP. For node $A$ the number of PoSW line cards, with symmetric communication assumption, is equal to:

$$
N_{P o S W}(A)=\left\lceil\frac{\sum_{0 \leq j<n, j \neq A} T(A, j)}{C_{P o S W}}\right\rceil,
$$

where $T(A, j)$ denotes the communication capacity between IPPoP nodes $A$ and $j$, and $C_{P o S W}$ the capacity of a PoS interfaced IPPoP to a WDMPoP line card.

The egress traffic from IPSDs to WDMPoP is aggregated on IPPoP to WDMPoP links as shown in Fig. 7 and Fig. 8. The aggregation is performed in the electrical domain on the packet level after the ingress optical-electrical (O-E) conversion and before the egress electrical-optical (E-O) conversion. OPhy in Fig. 8 denotes the optical physical layer. 
Fig. 7 Traffic aggregation in router
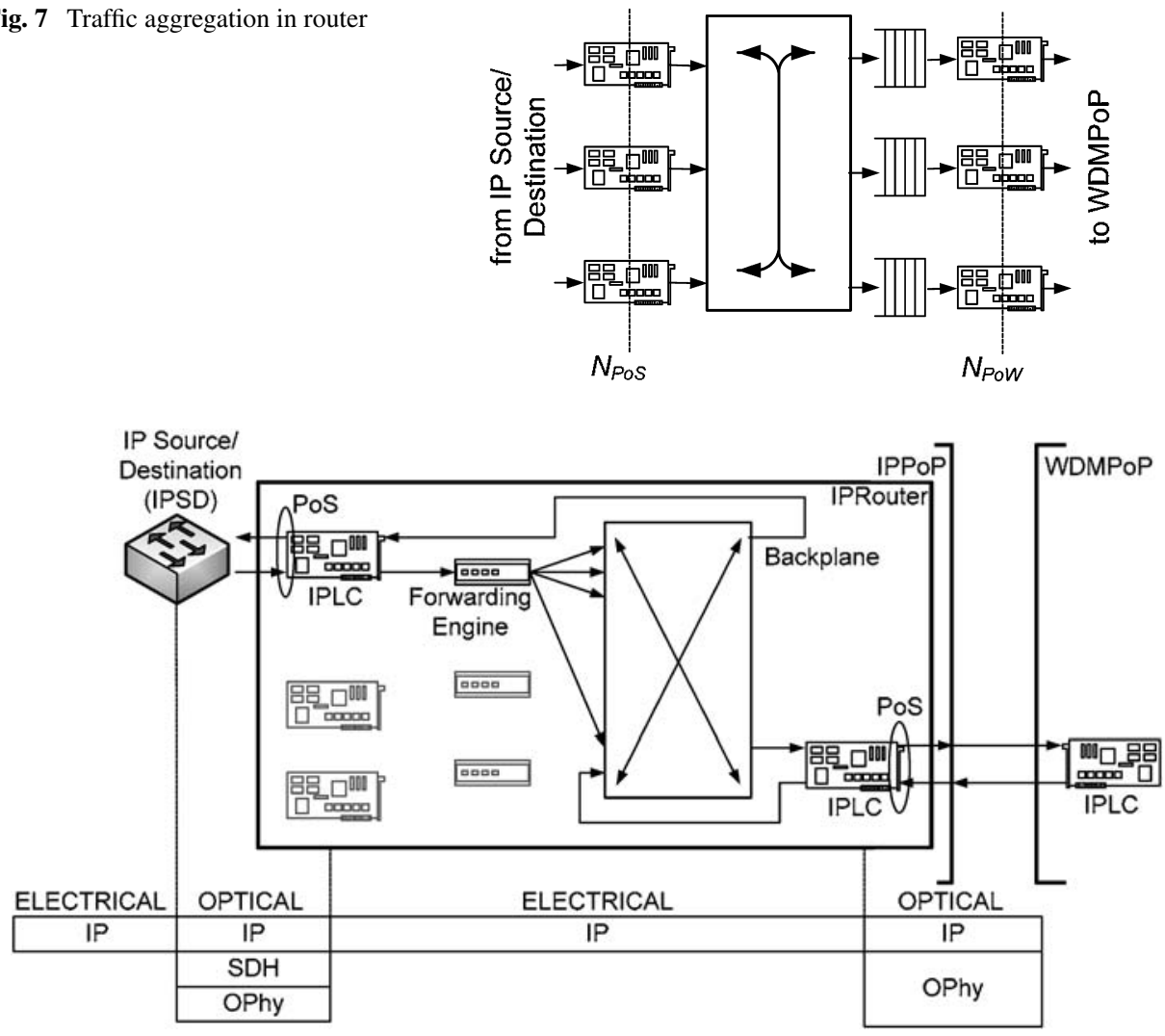

Fig. 8 IPPoP /WDMPoP communication

A packet arrives from the PoS interfaced card to the forwarding engine module which forwards the packet to a correct backplane input and eventually to one of the egress First-In-First-Out (FIFO) organized electrical buffers. Each buffer corresponds to one PoSW card, or to one baseband IPPoP - WDMPoP connection. The forwarding choice is based on the buffer states which are examined on each packet arrival. The buffer with the shortest packet queue (smallest number of buffered packets) is chosen. This algorithm assures that the input traffic does not overflow the IPPoP-WDMPoP connections, and balances the load among all access links. Figure 8 depicts the interconnection of described modules in the router module.

\section{Optical packet}

The packet based communication resembles burst switching networks, with the distinction that the control data are being sent along with the payload, unlike the separate control channels present in the burst based communication. The packet communication enables simplification of the edge equipment and implies substation of the IP terabit routers by the core optical cross-connects.

In order to reduce the number of entities to be processed in a given period of time, one or more client packets are grouped according to a selected criterion, like the common destination or the grade of service. Optical packet payload is formed out of aggregated client packets. Packets of the 


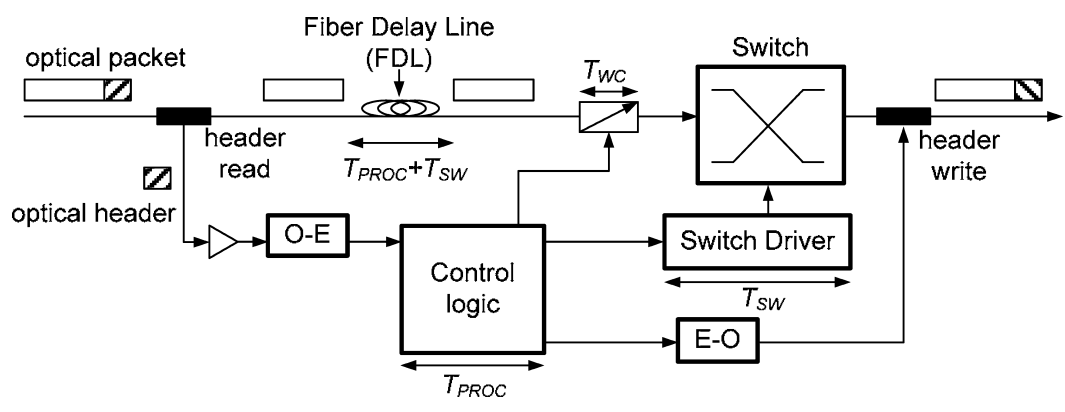

Fig. 9 Optical packet related actions

constant length (usually short) are often determined as cells. The cell communication reduces complexity of the packet switch, but increases complexity of the user-network interface. The most complex aspect of the cell communication is the implementation of the real-time packet scheduling mechanism using electronic components.

The packet header is being read in the electrical domain preceded by the opto-electrical conversion. The routing mechanism has to be simplified in order to prevent longer delays of packet payloads during the decision process. The proposals of routing in the optical domain are hence mostly based on labels and Multi-Protocol Label Switching (MPLS)-like switching. A packet label is replaced with a new one, and the packet is sent to the next interface according to the old label. However, some electrical features, like the label stacking, are difficult to implement in optical networks.

Packet headers are sent in series with the packet payload using the same transmission speed (IP datagrams, ATM cells). Header data processing in electronic routers or switches is conducted at the same speeds on which the payload data is received. This approach is difficult to implement in optical domain due to much higher transport capacities than in the electronic domain. The electronic manipulation of the header data, often assumed in optical networks, cannot cope with such high line speeds, making "on-the-fly" packet switching impossible. This problem is solved by creating an artificial payload delay (temporary buffering of the payload data), while the control mechanism performs O-E-O conversions, label analysis and switching decision. Figure 9 depicts actions in the WDMPoP node related to optical packet header reading, writing and component setup.

Optical packet header is being read and processed in the control logic. This procedure takes time $T_{P R O C}$, as it can include routing table consultation and selection of the output $\{$ link, wavelength $\}$ set. Based on the extracted information and the routing decision, control mechanism sets the configuration of the space switch using the switch driver. This procedure takes time $T_{S W}$, implying that the packet has to be delayed for time $T_{P R O C}+T_{S W}$. It is assumed that the wavelength converter setup time $T_{W C}$ takes less than the switch matrix reconfiguration. Header of the delayed packet can be altered when the packet arrives to the appropriate output on the appropriate wavelength (e.g. if label swapping instead of explicit addressing is used, or if the hop count is included in the header).

Figure 10 depicts a generic format of the optical packet, with the following parts:

- Optical Guard Band (OGB) assures that consecutive packets do not overlap in the optical cross-connect. The packet time sliding introduced by the components, transmission, switching or header writing time variation is reduced or eliminated using this gap, 


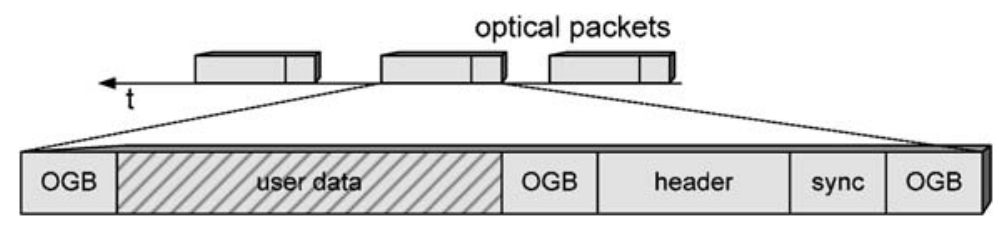

Fig. 10 Optical packet structure

- Synchronization time (sync) marks the beginning of each optical packet. This field is essential for the asynchronous communication, while in synchronous networks it serves for time sliding compensation. Synchronization time enables resynchronization of local time clock in both approaches,

- Optical packet header is generally shorter than the IP header and is usually denoted as label. Header usually contains the address of the destination node, packet priority, the information whether the packet carries some user data, packet length, and hop count. Packet length is necessary for asynchronous networks.

\section{A. User data modelling}

User data in the optical packet contains client packets from the photonic layer client. Those are usually IP packets, but it can be any other form of user data (e.g. ATM cells) as OPS is user data format transparent. One of the important factors when modeling aggregation of user data into optical packets is the selection of distribution of client packet lengths. In this work we have focused on IP packets and the empirical model obtained by measurements in year 2002 [6]:

- Average packet length is 402.7 bytes,

- Roughly $35 \%$ of packets has the length of 40 bytes as they correspond to session initiating Transmission Control Protocol (TCP) packets,

- The other typical packet length is 576 bytes (the traditional maximum segment size value),

- Packets of 1500 bytes (Ethernet length of the maximum transfer unit) make $10 \%$ of the total packet count, but $37.1 \%$ of the total network load.

Mentioned data were taken into account during packet generators' parameterization in order to achieve more accurate performance calculation. One of the most simple packet generators is based on the standard Poisson process with exponentially distributed packet lengths with mean value usually set to 500 bytes. A probability density function of the distribution defined in [6] had to be determined to generate packets whose lengths resemble to those measured in the real networks. Figure 11 depicts the obtained probability density function, while discontinuations represent steps on certain packet lengths which are often present (e.g. 40, 576 or 1500 bytes).

One of the approaches to packet length generation is the polynomial approximation (a polynomial fit) of the probability density function (PDF), followed by the extraction of the function roots (packet lengths) in randomly selected points (probabilities). Figure 11 depicts the 6-th order polynomial approximation. Described procedure would be too time consuming for calculation on the packet basis. It is easier to make the approximation of the inverted PDF function using straight line segments (Fig. 12). The input data are randomly generated numbers (with uniform distribution). The calculation of the packet lengths follows the selection of the region (straight line segment) which contains the randomly generated number.

Figure 13 shows the histogram of obtained packet lengths. Each bar corresponds to the number of packets whose length is between the values left and right from the bar. 


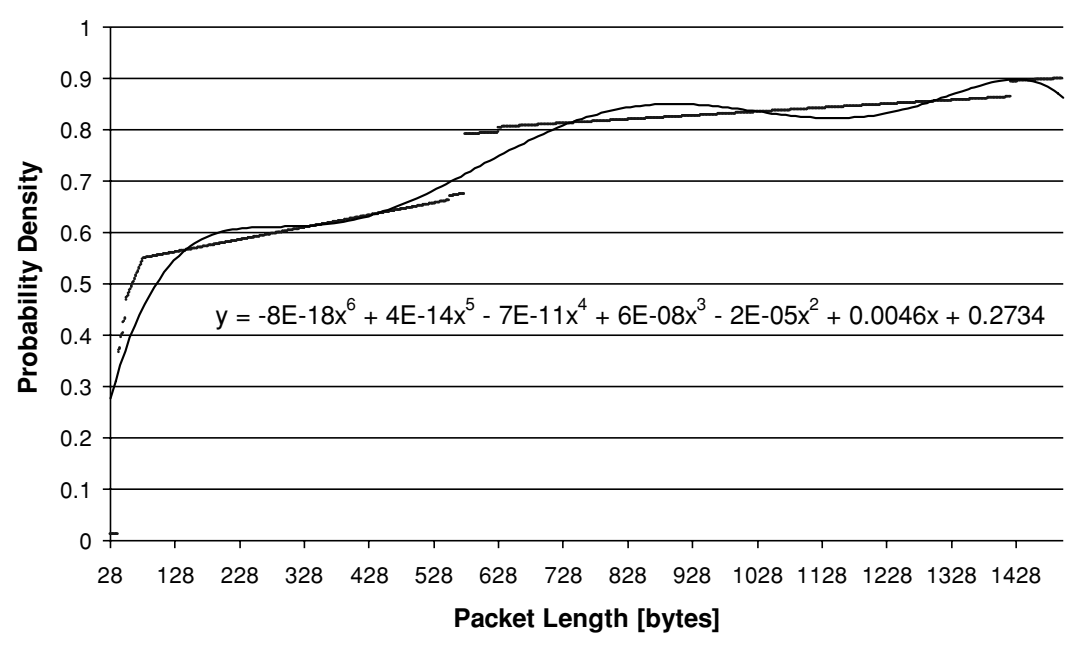

Fig. 11 6-th order polynomial approximation of the probability density

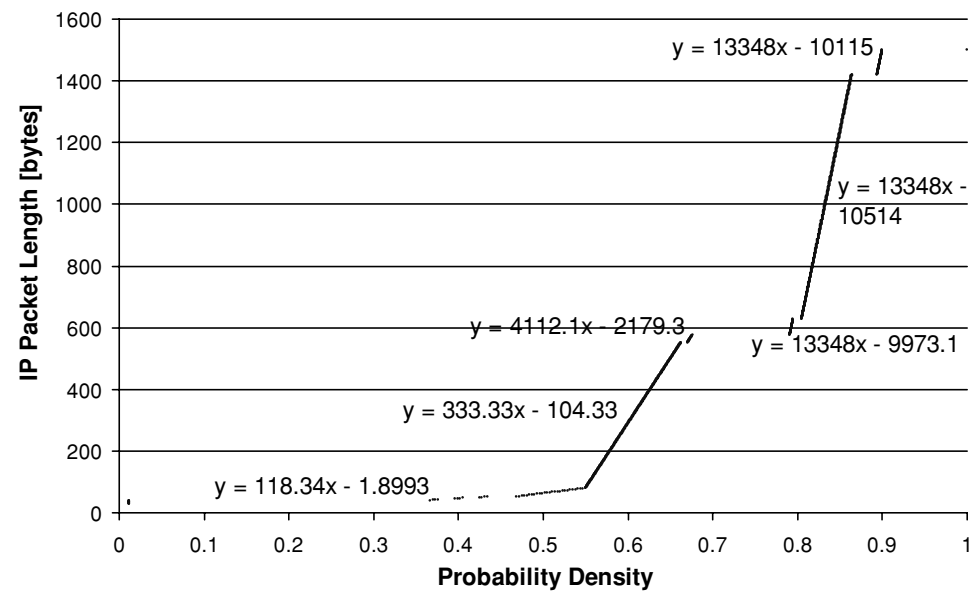

Fig. 12 Approximation of the inverted probability density function

The described approach to packet length calculation has to be further integrated with the selfsimilar traffic generation. Self-similarity is in this case related to calculating lengths of the packet bursts that are to be generated. After the length of the burst to be generated has been calculated, actual burst is being generated by generating individual packets within the burst, until the total generated packet size does not exceed the burst size. Packet lengths are generated using the uniform random number generator and the obtained approximation of the inverted probability density function. 


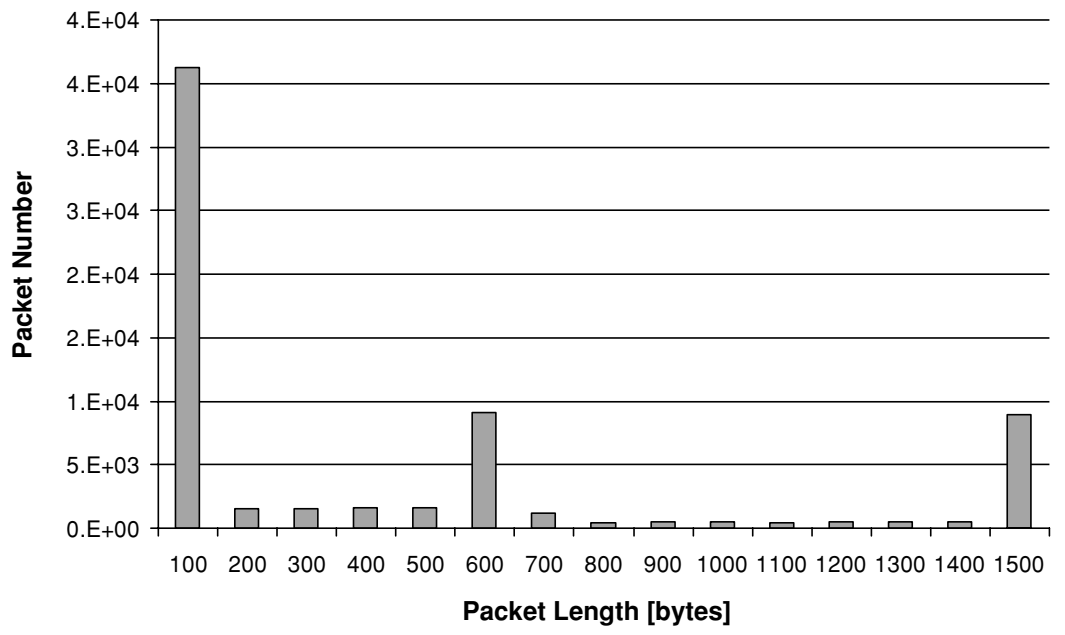

Fig. 13 Histogram of generated packet lengths

\section{Assembler}

A sort of aggregation mechanism is needed to store and classify input IP packets to form payloads for optical packets. Besides the payload formation, the aggregation also shapes input traffic and eliminates high variability introduced by the self-similarity [7]. Optical burst switching (OBS) assumes the input aggregation to form bursts [8] which are, in general, longer than optical packets. The difference between the OPS and OBS hence arises more in the core than in the access nodes of the optical network, as the OBS network requires configuration of core switches by a signaling protocol prior to the data exchange.

The assembler module performs the aggregation of client packets along with the contention resolution based on delaying locally generated packets [9]. General assembler structure is shown in Fig. 14. It includes line cards, aggregator, disaggregator and holder module.

The communication between the assembler and the IPPoP node is performed using the same number of PoSW line cards in the assembler as in the IPPoP node. The cards supporting the communication between assembler and the other parts of the WDMPoP node do not utilize the PoS interfaces, but the packet over wavelength (PoW) interfaces. From the broader functional perspective on the WDMPoP level, assembler performs the conversion between input PoS optical signal (used in optical circuit switched network) to packet over wavelength optical signal (used in optical packet switched networks). The PoW interfaces in this work substitute future commercial solutions for IP-over-WDM communication, which does not utilize a layer 2 protocol (like Sonet/SDH), but uses a simple framing protocol for synchronization and packet delimitation.

Figure 15 depicts a simplified aggregator and Fig. 16 a simplified holder scheme. Aggregator stores ingress IP packets to collect enough data for the optical packet payload. The input packets are sorted according to their final destination as one optical packet can transport only several IP packets with the same destination (IPPoP node). This is implemented in the sub-module denoted as sorter.

The packet classification and buffering is performed in the electrical domain, as input packets arrive after opto-electrical conversion and deframing in PoSW cards. The FIFO organized buffers are time and length constrained. Data is collected (packet are buffered in the aggregator) until some maximum data size for one optical packet is acquired (MPS - Maximum Packet Size), or 


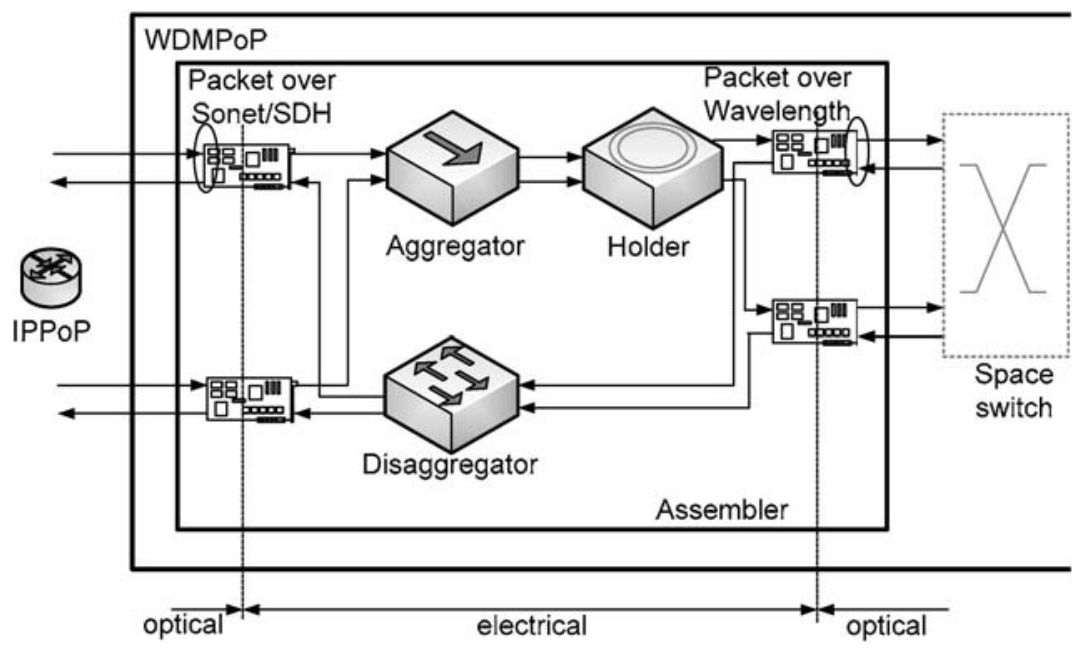

Fig. 14 Assembler structure

Fig. 15 Aggregator structure

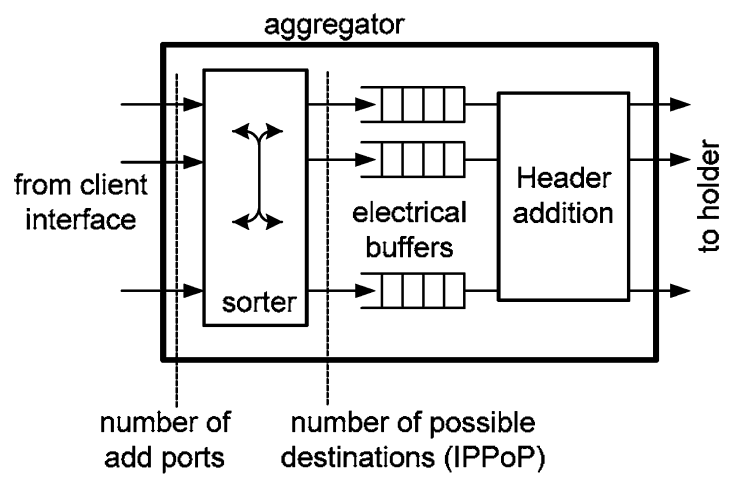

Fig. 16 Holder structure

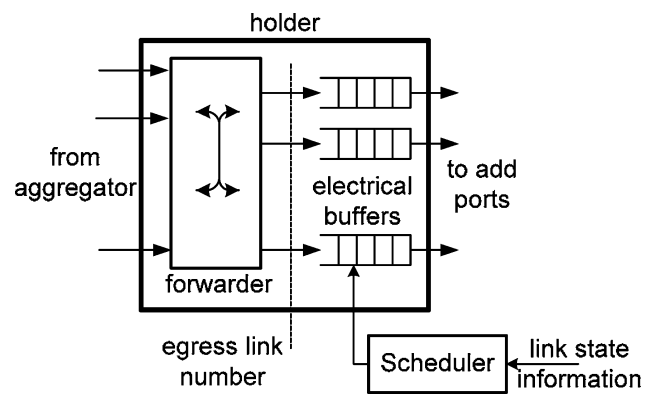

until the packets are being buffered longer than some maximum time limit. In the case of the timer expiration, the packet load of the created optical packet is shorter than the MPS.

Aggregated data serves as the payload for optical packet created in PoW interfaced cards. Each card is connected to a transponder which adjusts the power level and assigns a correct wavelength to a signal. The wavelength is determined by the state of the output port (channels on appropriate output link). If all the channels are occupied, the wavelength is determined by 
the state of buffer on the selected output if the buffering is used as the contention resolution mechanism.

In order to reduce the buffer occupancy created by the locally generated packets and hence decrease the packet loss probability of transit packets, the creation of the optical packet can be delayed until one of the appropriate egress channels becomes available. This delay is optional and can be introduced in the holder module adjacent to the aggregator module. The buffering principle is the same as in the aggregator, but the number of buffers differs, as it equals the number of egress links in the holder case. Ingress data is forwarded to the appropriate buffer by the module denoted as forwarder. The transparency of the network is preserved, as the possible input delays in buffers occur while the aggregated loads for optical packet are still in the electrical domain.

\section{A. Aggregation of IP datagrams and creation of optical packets}

IP network (e.g. LAN) represents the optical network client and serves as the source of IP packets. IP packets are delivered to the optical network by baseband transmission (usually Sonet/SDH frame), where they form user data of the optical packets. One optical packet contains one or more IP packets with the same destination, or a common part of the path if the optical packet content change is allowed in the intermediate nodes. Optical packets are created in the aggregator in the edge nodes.

Figure 17 depicts the block scheme of the aggregation mechanism. It is assumed that the aggregator maintains a separate electronic buffer for each possible destination (i.e. IP/WDMPoP node). We will denote the buffer associated with the destination $i$ as $q_{i}$. Aggregator is parameterized by two values - maximum (optical) packet size (MPS), and maximum (allowed) aggregation time (MAT) [7]. MPS determines the upper length constraint, and MAT the upper time constraint of the aggregation mechanism. Buffer $q_{i}$ is parameterized by the buffer length $S\left(q_{i}\right)$, and the timer value $T_{i}$. The timer measures the elapsed time of the current aggregation.

\section{Results}

The analyzed network topology corresponds to the reference network denoted as the core network within the COST 266 project [10], and depicted in Fig. 18. The traffic capacities between IPPoP pairs were generated using the enhanced population-distance model [10]. The year 2004 traffic volumes over 4 wavelengths and $40 \mathrm{Gbps}$ channel system were assumed. Each node output has been equipped with a fiber delay buffer, with 3 fiber delay lines. The base fiber delay line has been constructed to correspond to 0.2 mean length packet durations. We have selected such dimensioning for this particular traffic setup using the simulation based implementation of variable length packet buffering performance analysis proposed in [11]. The fiber delay line buffer depth influences the absolute result values, but do not change the obtained trend of results. Additionally, full wavelength conversion is assumed in each node.

The generated traffic demands were scaled in a way that the highest link load corresponds to the chosen network load.

The aim was to determine the influences of the aggregation parameters on the packet loss probability and total introduced delay and the influence of the used holder mechanism on the packet loss probability. We have varied maximum link load in the network in the range $0.5-1$ erl, what scales the loads of all links in the network, so that the load of the most heavily loaded link corresponds to the chosen load. It is assumed that all wavelengths can be used for OPS communication, and that the full wavelength conversion is employed. These assumptions enable 


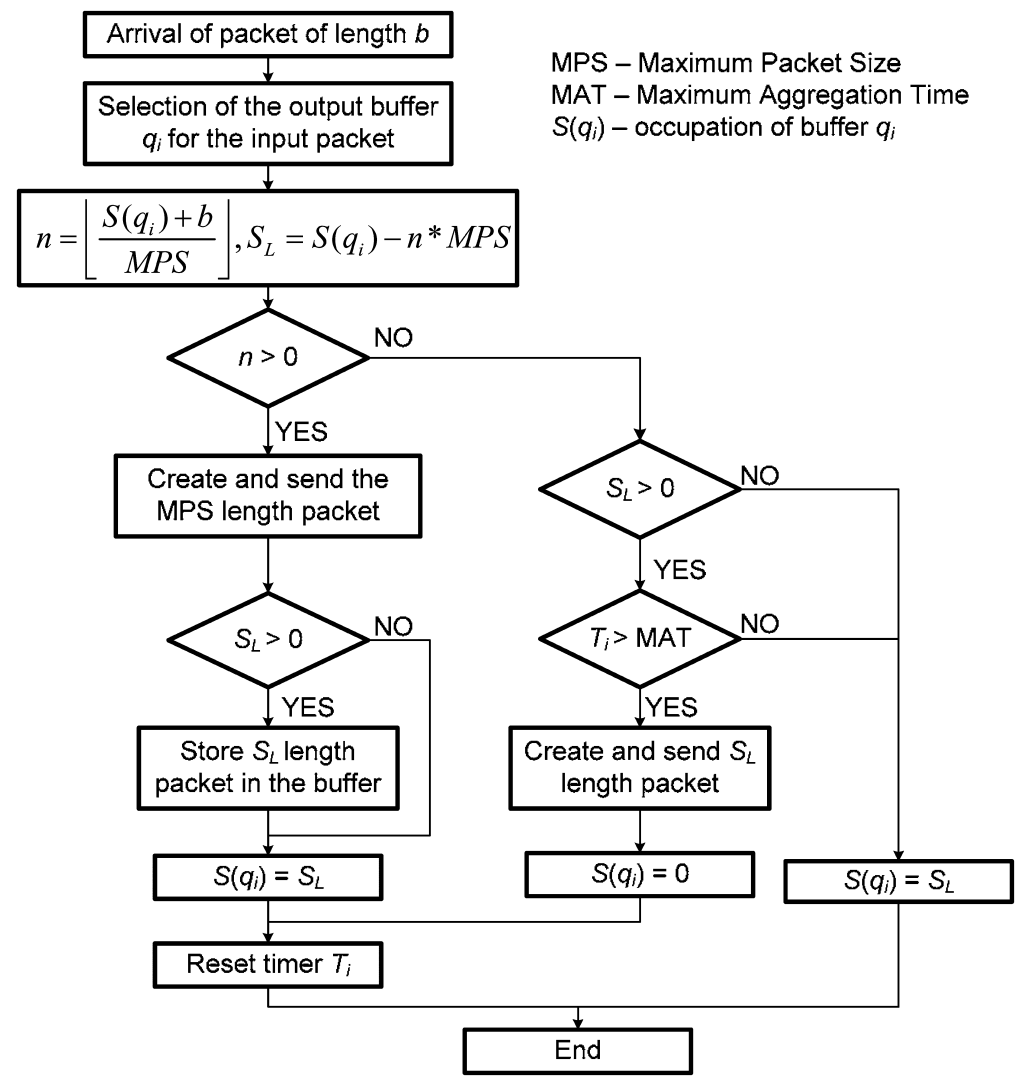

Fig. 17 Block scheme of the aggregation mechanism

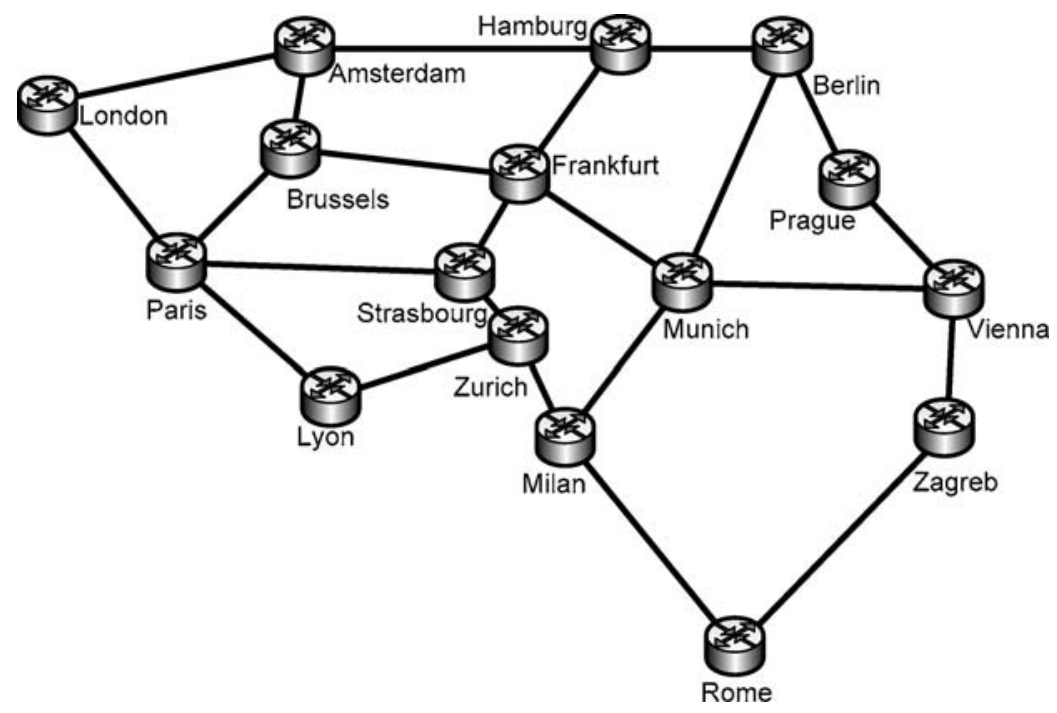

Fig. 18 Analyzed network topology 


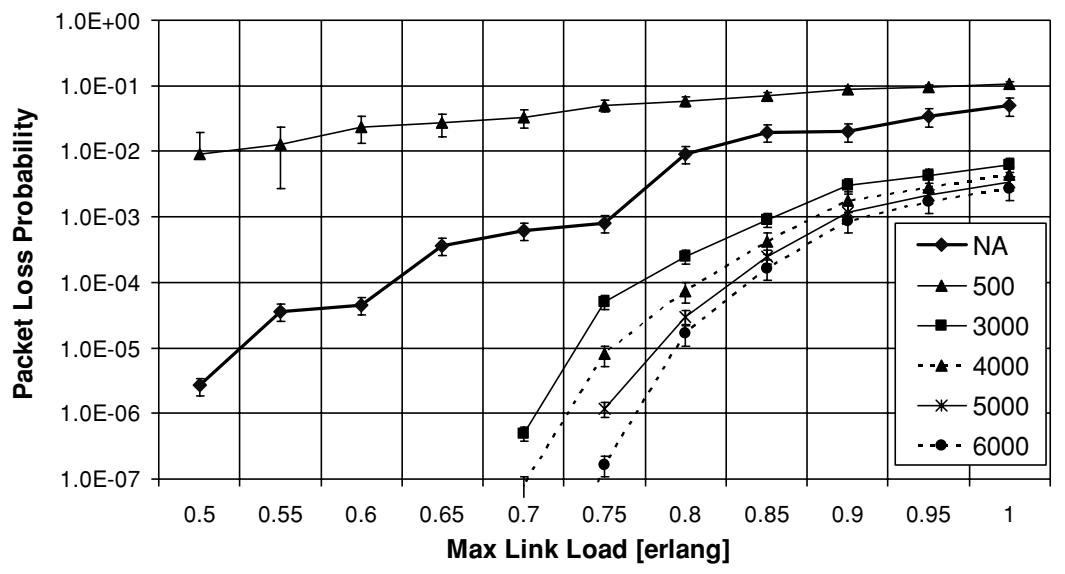

Fig. 19 Dependence of the packet loss probability on the maximum link load

simple implementation of load balancing procedure among wavelengths of the same fiber. Similar traffic loads on all wavelengths imply that the link load can be expressed as the mean value of wavelength loads.

A discrete event simulation with self-similar traffic generator based on the fractional Gaussian noise was used [12]. The used packet length probability density function corresponds to the one described in [6] in order to imitate the Internet traffic.

\section{A. Aggregation mechanism}

Figure 19 shows the influence of the aggregation on the mean Packet Loss Ratio (PLR) of all network demands. A demand defines communication between two IPPoP nodes. The MAT value has been set to 100 mean packet durations (duration of the $100 \times 402.7$ bytes on the line speed). Each curve corresponds to one MPS value. Label NA denotes the case without aggregation, where the input IP packets are packed into optical packets as they arrive. Other MPS values are expressed in bytes. Packet loss probability is calculated for each maximum link load constraint change. Some authors tend to denote this constraint as the network load.

The thick line depicts the packet loss probability without the input aggregation, implying that the IP packets were packed into optical packets as they arrive. With the increase of MPS from 3000 to 6000 bytes the packet loss probability decreases. The value of 500 bytes is considered just for comparison purposes as it represents the fragmentation of input packets in most cases.

The results indicate that the packet loss probability decreases with the increase of the maximum packet size. In other words, communication will have lower packet loss probability if longer optical packets are used. Generally speaking, this result can be justified taking into account the fact that a packet contention will occur if all available output resources (links) are occupied at the moment a new packet arrives. Shorter maximum packet size in combination with arrival of potentially long bursts can cause generation of many short packets that will be sent to the network all at once, or overlapping in time. Such parallel packets will use in parallel all available wavelengths, and increase the probability of contention in all subsequent nodes. Such overlapping will be shorter (or eliminated) as the maximum packet size is longer, because one burst will be transferred using fewer packets. Longer maximum packet sizes are hence more appropriate for the 


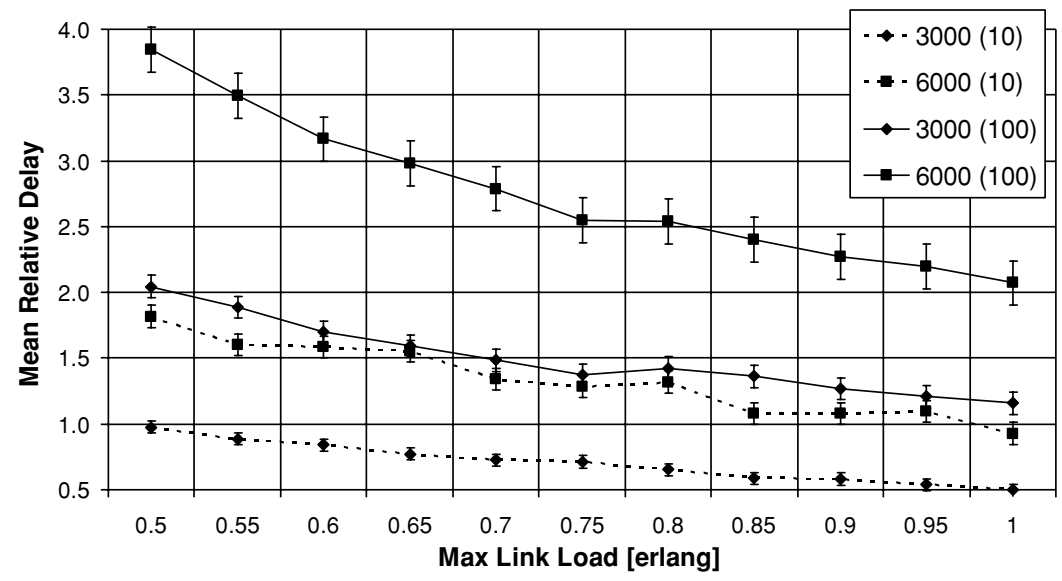

Fig. 20 Dependence of the relative mean delay on the maximum link load

arrival of client (IP) packets in bursts generated by self-similar process. Moreover the variability of the input self-similar traffic is reduced by traffic shaping introduced by aggregation.

The next group of results focuses on dependence between mean delays introduced due to aggregation and the mean duration of the IP packet on the selected line speed, as depicted in Fig. 20. The delay is calculated as the mean value of delays for the end-to-end communication between all IPPoP pairs. The figure depicts delays for MAT $=10$ (dotted line) and MAT $=100$ (solid line), and for the set of MPS $=\{3000,6000\}$. It is clear that the delay is increasing by the increase of MAT and MPS, but is decreasing with the maximum link load increase, as the MAT length of the optical packet is achieved more quickly due to more frequent packet arrivals.

Figure 21 shows maximum introduced packet delay assuming mentioned network setup. The delay corresponds to the maximum delay among all end-to-end communications between all IPPoP pairs. The solid line denotes two MPS setups for MAT $=100$, while dotted line denotes two MPS setups for MAT $=10$, being MPS $=3000$ and MPS $=6000$. The MPS value generally does not have a clear influence on the maximum delay. This (un)correlation can pose difficulty for the service provision with the delay variation guarantees.

Relative reduction of the packet loss probability (gain) is depicted in Fig. 22. The increase of the maximum link load reduces the algorithm efficiency and reduces the differences between the best MPS $=6000$, MAT $=100)$, and the worst case (MPS $=3000$, MAT $=10)$. All other gains for different MPS and MAT values between those two extreme cases are situated in between the depicted gains.

\section{B. Holding mechanism}

The second group of results focuses on the holder influence on the packet loss probability and the delay the mechanism introduces. Figure 23 depicts packet loss probability with and without holder. The used buffers in the holder were ideal without capacity limitation.

The results have a good match on lower maximum link loads, but they differ on the loads above $0.5 \mathrm{erl}$. The worst case without holder has the linear increase of the packet loss probability, while the worst case with holder has almost negligible increase on loads above 0.6 erl. This is the consequence of the fact that almost all add traffic can be buffered in the case of congested network as the buffers are limitless. 


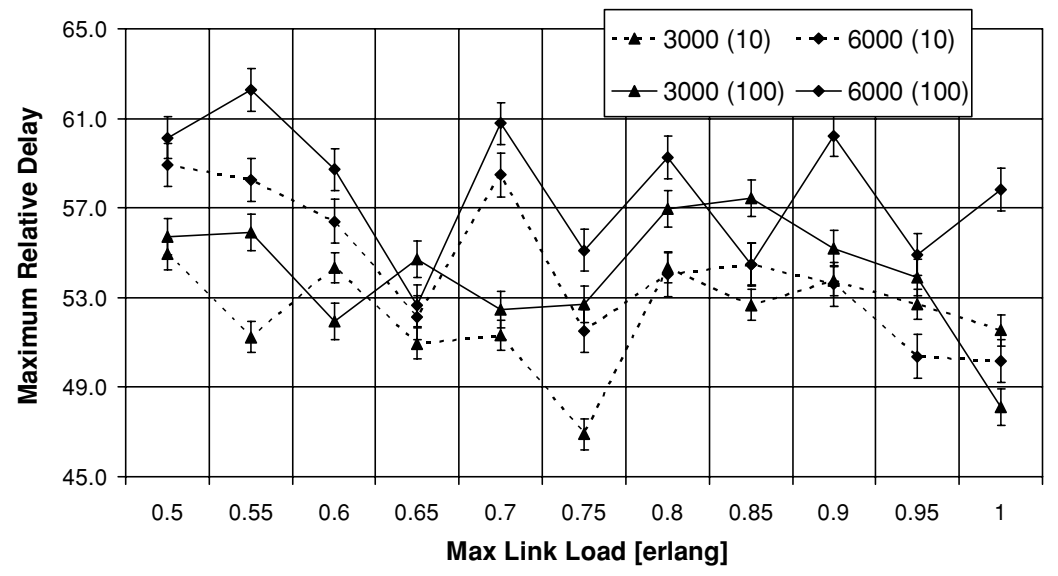

Fig. 21 Dependence of the relative maximum delay on the maximum link load

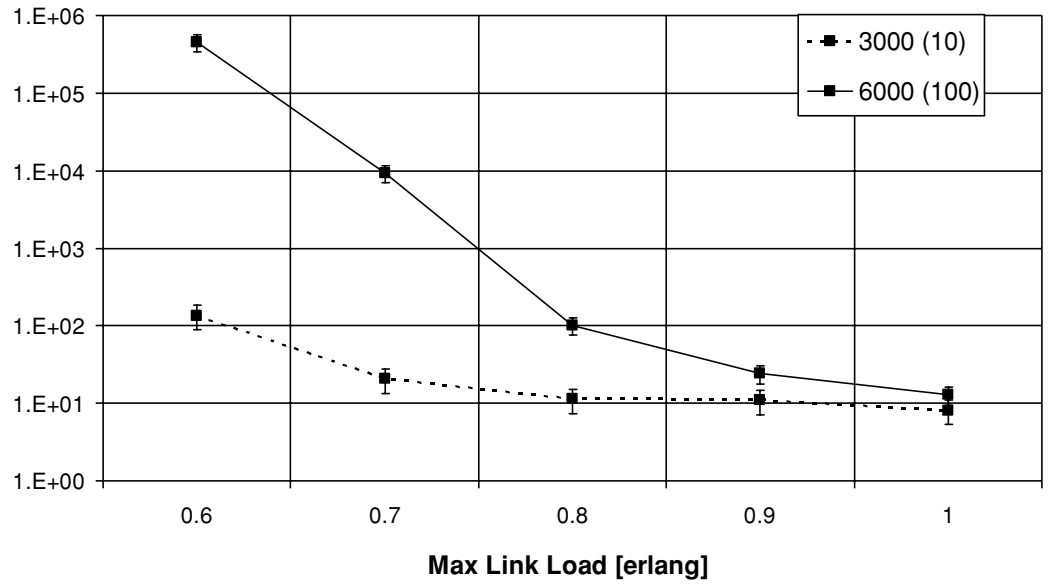

Fig. 22 Dependence of the relative packet loss probability reduction on the maximum link load

The buffering introduces a considerable delay as depicted in Fig. 24. Delay time is calculated as the mean delay among all generated packets, includes total delay in the access part (i.e. caused by packet aggregation and holding), and is normalized to the duration of the IP packet with mean load. Delay time has been shown in the case of a holder with unlimited buffer size, as well as with limited buffer size equal to 1000 mean IP packet lengths. These results are accompanied by the number of rejected packets due to buffer overflows in the second case.

With the load increase the delay increases in the ideal case of unlimited holder buffers, due to the increase of the number of buffered packets. In the second case a limited holder can successfully buffer almost all packets up to $0.5 \mathrm{erl}$ of load that would be otherwise buffered in the FDL buffer, while the rejection increases rapidly with larger loads. The introduced delay is reduced with the increase of the maximum link load in both cases as the optical packets are created faster due to higher input packet arrival rates. Holder does not have a large influence on this delay on lower link loads, as the optical packets can be sent to output links immediately if free resources are found. With the link load increase the delay introduced in holder increases, 


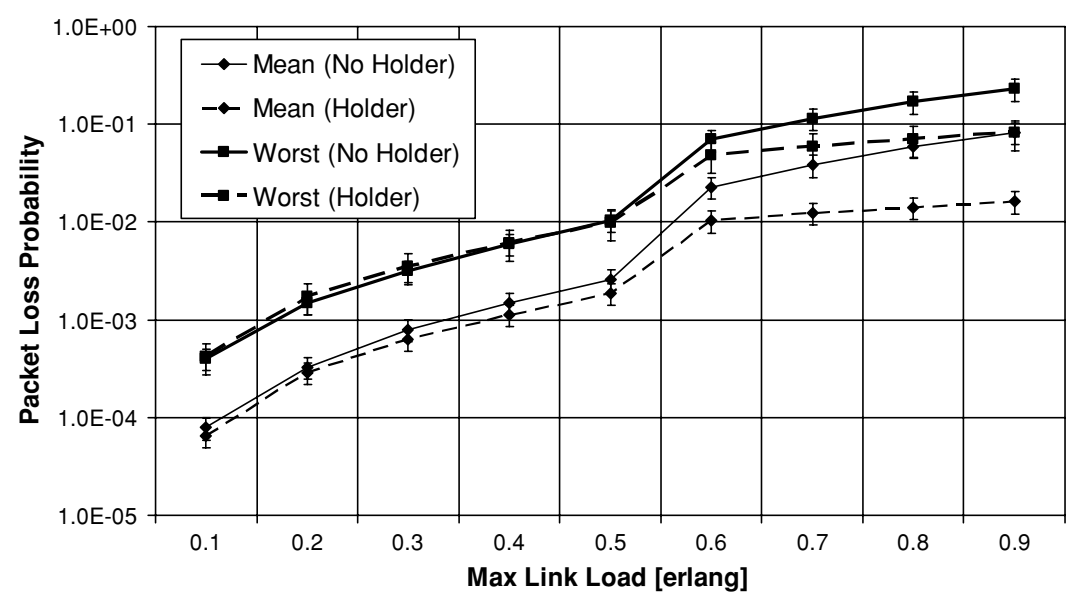

Fig. 23 Influence of the input packet hold on the packet loss probability

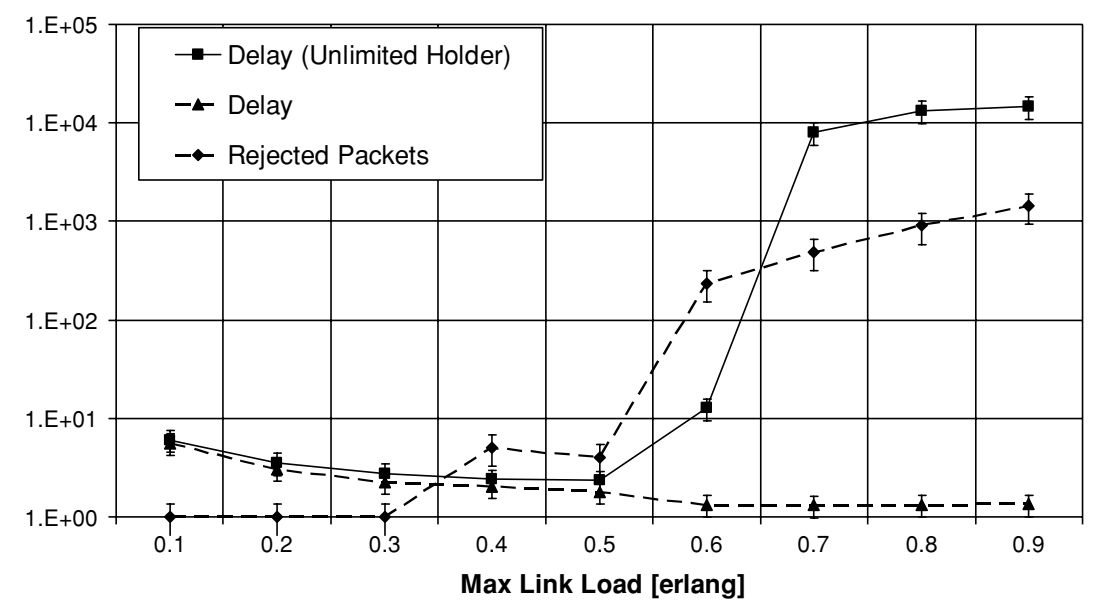

Fig. 24 Dependence of the normalized delay time and the number of rejected packets on the holder structure and the maximum link load

but it is cancelled out by the decrease of the aggregation delay. The differences between delay results arise on link loads larger than $0.5 \mathrm{erl}$, as the packet delay continues to decrease in the limited buffer size with the further increase of the link load, while it starts to increase in the case of the unlimited buffer size. In the first case the buffers in holders become full and start to reject new packets due to overflow. In those circumstances the introduced delay in holder buffers does not further increase, while the aggregation delay is further reduced as the input packet arrival rates increase, causing the total delay to decrease. In the case of the unlimited buffer, the delay introduced in holder increases more rapidly than the aggregation delay decreases what results in total delay increase. 


\section{Conclusion}

The IP to photonic layer interface has to be carefully constructed as it can reduce the packet loss probabilities in the packet switched optical network and increase the offered quality of service. The optical network model, along with the models of IPPoP and WDMPoP network nodes, has been proposed. As a part of node and network performance evaluation the influence of the interface part of the WDMPoP node on the packet loss probability has been analyzed. The interface has been modeled as the assembler module performing input aggregation of the IP packets in order to form optical packets and the input delay of aggregated data as a form of contention resolution. Used network and traffic data were based on the COST 266 project, while the traffic sources followed the fractional Gaussian noise model. IP packet lengths have been modeled using empirical data.

The results show that the increase of the maximum optical packet size and the input delay can reduce the packet loss probability. These results are not to be taken as a general conclusion as they reflect the influence of those two mechanisms alone on the OPS based network performance. The increase of the packet size is limited by the switch performance and the delay line lengths in the buffers, while the holder introduces input delay which can be unacceptable for certain requests with quality of service guarantees.

Acknowledgment This work has been conducted with the support of the Swiss Federal Office for Education and Science within the European Actions COST 266 and COST 291.

\section{References}

1. Yao, Shun, Biswanath Mukherjee, and Sudhir Dixit, Advances in photonic Packet Switching: an overview, IEEE Communication Magazine. (Feb. 2000) pp. 84-94.

2. Lackovic, Marko, and Robert Inkret, network design, optimization and simulation tool cosmos, in: Proc. of WAON, Zagreb, Croatia. (June 13-14) (2001) pp. 37-44.

3. Yao, Shun, Biswanath Mukherjee, S.J. Ben Yoo, and Sudhir Dixit, A unified study of contention-resolution schemes in optical packet-switched networks, IEEE Journal of Lightwave Technology. (Mar. 2003) pp. 672683.

4. Callegati, Franco and Walter Cerroni, Wavelength allocation algorithms in optical buffers, in Proc. of IEEE International Conference on Communications, ICC 2001, vol. 2 (June 11-14 2001) pp. 499-503.

5. Callegati Franco, Giorgio Corazza, and Carla Raffaelli, Exploitation of DWDM for Optical Packet Switching With Quality of Service Guarantees, IEEE Journal on Selected Areas in Communications. 20(1) (Jan. 2002) pp. 190-201.

6. http://advanced.comms.agilent.com/insight/2001-08/TestingTips/1MxdPktSzThroughput.pdf, Agilent Technologies, 2001 (last accessed 23/02/2006)

7. Xue, Fei, Shun Yao, Biswanath Mukherjee, and S.J. Ben Yoo, The performance improvement in optical packet-switched networks by traffic shaping of self-similar traffic, in Proc. of OFC 2002 .(Mar. 2002). pp. 218-219.

8. Ge, An, Franco Callegati, and Lakshman S. Tamil, On optical burst switching and self-similar traffic, IEEE Communication Letters. 4(3) (Mar. 2000) pp. 98-100.

9. O’ Mahony, J. Michael, Dimitra Simeonidou, David K. Hunter, and Anna Tzanakaki, The application of optical packet switching in future communication networks, IEEE Communication Magazine. (Mar. 2001) pp. 128-135.

10. Inkret, Robert, Anton Kuchar, and Branko Mikac (eds), Advanced Infrastructure for Photonic Networks, Extended final report of COST 266 Action, University of Zagreb, Faculty of Electrical Engineering and Computing, Zagreb, Croatia, 2003.

11. Callegati, Franco, Optical Buffers for Variable Length Packets, IEEE Communication Letters. 4(9) (Sept. 2000) pp. 292-294.

12. Lackovic, Marko, Branko Mikac, and Vjekoslav Sinkovic, Network Performance Evaluation by Means of Self Similar Traffic Model, in Proc. of Mipro. Opatija, Croatia, (May 19-23, 2003) pp. 82-87. 